\title{
Live birth rates and safety profile using dydrogesterone for luteal phase support in assisted reproductive techniques
}

Ravichandran Nadarajah ${ }^{1}$, MBBS, MRCOG, Hemashree $\underline{\text { Rajesh }}^{1}$, MBBS, MRCoG, Ker Yi Wong ${ }^{1}$, BEng, MD, Fazlin $\underline{\text { Faisal }}^{1}$, MBBS, Su Ling $\underline{Y u^{1}}$, MMed, FRCOG

INTRODUCTION Assisted reproductive techniques (ARTS) result in a deficient luteal phase, requiring the administration of intramuscular, intravaginal or oral exogenous progesterone. Dydrogesterone, an oral retroprogesterone with good bioavailability, has been used in assisted reproductive cycles with outcomes that are comparable to those of vaginal or intramuscular progesterone. However, there are limited reviews on its use for luteal phase support in ARTs, in terms of pregnancy outcomes and associated fetal anomalies. This study aimed to review the live birth rates and associated fetal anomalies of women who were given dydrogesterone for luteal phase support in assisted reproductive cycles at a tertiary hospital in Singapore.

METHODS This retrospective descriptive study included 1,050 women who underwent in vitro fertilisation/intracytoplasmic sperm injection at the Centre for Assisted Reproduction of Singapore General Hospital between 2000 and 2011 . The women were given dydrogesterone for luteal phase support. The main outcome measures were rates of pregnancy, live birth, miscarriage and fetal anomalies.

RESULTS The pregnancy and live birth rates were $34.7 \%$ and $27.7 \%$, respectively. Among those who achieved pregnancy, $17.0 \%$ miscarried, $0.8 \%$ had ectopic pregnancies and $0.3 \%$ had molar pregnancies. Fetal anomalies were detected in $1.9 \%$ of pregnancies, all of which were terminated by choice.

CONCLUSION Since the outcomes of dydrogesterone are comparable to those of intramuscular and vaginal progesterone, it is a reasonable option to provide luteal phase support for women who are uncomfortable with injections or vaginal insertions. Randomised controlled studies are needed to determine the optimal dosage of dydrogesterone for luteal phase support in ARTs.

Keywords: dydrogesterone, fetal anomalies, live birth, luteal phase support, pregnancy

\section{INTRODUCTION}

Assisted reproductive techniques (ARTs) are known to produce a deficient luteal phase, which is associated with pregnancy failures. ${ }^{(1-3)}$ Various mechanisms, such as the downregulation of pituitary function due to the use of gonadotropin-releasing hormone agonists and the direct inhibition of luteinising hormone by high steroid levels resulting from multiple corpora lutea, have been proposed to explain the aetiology of luteal phase deficiency in ARTs. ${ }^{(4)}$ Both progesterone and human chorionic gonadotropin (hCG) supplementation have been used for luteal phase support in ARTs, with improved fertility outcomes when compared to non-supplementation. ${ }^{(5,6)}$ In a recent Cochrane review, ${ }^{(6)}$ progesterone was recommended as the agent of choice for luteal phase support; however, the use of hCG was strongly discouraged due to the increased risk of ovarian hyperstimulation syndrome.

Progesterone for luteal support can be administered intramuscularly, intravaginally or orally. Parenteral administration of progesterone (i.e. through the intramuscular or vaginal routes) has been widely used, as it results in rapid absorption and high serum concentrations. ${ }^{(7)}$ However, intramuscular applications can be painful, and vaginal applications have been associated with vaginal discharge and irritation. (7) Oral progesterone, although advantageous due to its ease of administration, results in poor outcomes when used for luteal support in ARTs. ${ }^{(8)}$ This is due to its poor bioavailability, as it undergoes rapid first-pass prehepatic and hepatic metabolism, which results in its degradation. ${ }^{(4)}$ Dydrogesterone, a synthetic retro isomer of progesterone, is also administered orally; however, it has better bioavailability than oral progesterone due to its enhanced configuration. Randomised studies by Chakravarty et $\mathrm{al}^{\left({ }^{(9)}\right.}$ Ganesh et $\mathrm{al}^{(10)}$ and Salehpour et $\mathrm{al}^{(11)}$ showed that the use of dydrogesterone resulted in pregnancy rates that were similar to those from the use of vaginal progesterone. In the randomised study by Chakravarty et al, ${ }^{(9)}$ live birth rates were also comparable between women who used dydrogesterone and those who used vaginal progesterone. To date and to the best of our knowledge, no studies have investigated live birth rates resulting from the use of dydrogesterone for luteal support in ARTs, other than the study by Chakravarty et al. ${ }^{(9)}$ This is despite the fact that live birth rates are a crucial concern for couples seeking fertility treatment. 
An overview of birth defects reported from 1977 to 2005 showed that the use of dydrogesterone during pregnancy was not associated with congenital malformations. ${ }^{(12)}$ However, there are limited reviews on fetal anomalies associated with the use of dydrogesterone for luteal phase support in ARTs. Thus, the present study aimed to review the pregnancy outcomes, namely the live birth rates and incidence of fetal anomalies, of a large cohort of women who were given dydrogesterone for luteal phase support in in vitro fertilisation (IVF) at a tertiary centre in Singapore.

\section{METHODS}

This was a retrospective study conducted at the Centre for Assisted Reproduction in Singapore General Hospital (SGH), a tertiary centre in Singapore. The study was approved by SingHealth's Centralised Institutional Review Board. Women who underwent fertility treatment in SGH from 2000 to 2011 were identified from the hospital database. The inclusion criteria were women who had undergone a fresh IVF/intracytoplasmic sperm injection (ICSI) cycle (non-donor cycle) and received oral dydrogesterone for luteal phase support. Women who were given progesterone intramuscularly or vaginally and those who were lost to followup or delivered overseas were excluded from the study. The final sample size was 1,050.

Each woman in the study underwent a tailored stimulation programme, using either an agonist or antagonist protocol, depending on the cause of their subfertility. Ovarian follicular development was monitored using transvaginal ultrasonography from Day 6 of stimulation. When three follicles had reached a maximal diameter of 18 mm, 10,000 IU hCG (Profasi; Serono, Geneva, Switzerland) was injected subcutaneously. Oocytes were retrieved transvaginally under ultrasonography-guidance 34-36 hours after hCG injection. Subsequently, either IVF or ICSI was performed, depending on the quality of the semen sample of the male partner. A maximum of three embryos were transferred on either Day 2 or Day 3 after retrieval, based on the quality of the embryos. Luteal phase support with dydrogesterone $10 \mathrm{mg}$ three times a day was started on the day of oocyte retrieval and continued till the 12th week of pregnancy.

Outcome measures were rates of pregnancy, miscarriage, live births and fetal anomalies. A viable pregnancy was defined as the presence of ultrasonography evidence of a fetal pole with fetal heart activity at six weeks. Miscarriage was documented when there was ultrasonography evidence of an intrauterine gestation $\mathrm{sac}>20 \mathrm{~mm}$ without a fetal pole and a crown rump length $>6 \mathrm{~mm}$ with absent fetal heart activity, or any other signs of pregnancy loss at $<24$ weeks of gestation. A live delivery at or after 24 weeks of gestation was counted as a live birth. First trimester screening (FTS) for trisomy 13, 18 and 21 was performed between 11 and 14 weeks of gestation, and fetal anomaly imaging was performed between 18 and 20 weeks of gestation. The women were offered amniocentesis or chorionic villi sampling if their FTS was returned as 'high risk' and if structural anomalies were detected in the fetal anomaly images. All babies were assessed by neonatologists at birth for gross anomalies.
Table I. Outcomes of the women in the sample population $(n=1,050)$.

\begin{tabular}{lc}
\hline Outcome & No. (\%) \\
\hline Did not achieve pregnancy & $686(65.3)$ \\
Achieved pregnancy & $364(34.7)$ \\
Live birth & $291(27.7)$ \\
Spontaneous miscarriage & $62(5.9)$ \\
Ectopic pregnancy & $3(0.3)$ \\
Molar pregnancy & $1(0.1)$ \\
Termination of pregnancy & $7(0.7)$
\end{tabular}

Table II. Types of fetal anomalies detected among the women who achieved pregnancy $(n=364)$.

\begin{tabular}{lc}
\hline Type of fetal anomaly & No. (\%) \\
\hline Anencephaly & $1(0.3)$ \\
Renal agenesis & $1(0.3)$ \\
Arthrogryposis & $1(0.3)$ \\
Cleft lip/palate & $1(0.3)$ \\
Exomphalos & $1(0.3)$ \\
Complex heart disease & $1(0.3)$ \\
Sacrococcygeal teratoma & $1(0.3)$ \\
Total & $7(1.9)$ \\
\hline
\end{tabular}

\section{RESULTS}

The sample population consisted of 1,050 women aged 21-45 (mean age 35) years. Table I shows the outcomes of the 1,050 women who underwent IVF/ICSI and received dydrogesterone for luteal phase support. Of these, $34.7 \%$ achieved pregnancy, while the remaining $65.3 \%$ failed to achieve pregnancy. Among those who achieved pregnancy, $79.9 \%$ delivered successfully, $17.0 \%$ miscarried spontaneously, $0.8 \%$ had ectopic pregnancies, $0.3 \%$ had molar pregnancies and $1.9 \%$ terminated their pregnancies. All the women who terminated their pregnancies did so due to the presence of fetal anomalies detected on fetal anomaly imaging. Among the 291 live births, none had any gross anomalies at birth detected by the neonatologists. Table II lists the types of fetal anomalies that were detected among the women who achieved pregnancy.

\section{DISCUSSION}

In ARTs, the use of progesterone in routine luteal phase support has achieved significantly higher pregnancy rates than placebos. ${ }^{(13,14)}$ Progesterone is currently available in intramuscular, vaginal and oral formulations, with the former two being the more widely used formulations. Intramuscular progesterone is rapidly absorbed by the body and reaches high serum concentration within two hours. ${ }^{(15)}$ Vaginal progesterone is available as tablet, gel or cream preparations. Compared to intramuscular progesterone, vaginal progesterone is cleared more rapidly from the body's circulatory system and requires higher dosages to achieve serum progesterone levels that are equivalent to that of a physiological luteal phase. ${ }^{(15)}$ In the Cochrane review of studies that compared the use of intramuscular and vaginal progesterone for luteal phase support, no significant differences 
were found between the two in terms of live birth and clinical pregnancy rates. ${ }^{(6)}$ While vaginal progesterone offers women a painless alternative, side effects such as vaginal irritation and discharge have been reported. (7)

Among the various formulations of progesterone available, the ease of administration of oral progesterone relative to vaginal or intramuscular progesterone is undeniable. However, the bioavailability of oral progesterone is only $10 \%$ that of intramuscular progesterone due to the hepatic first-pass effect. ${ }^{(16}$ Dydrogesterone, a retro isomer of progesterone, has an additional double bond between carbon- 6 and carbon-7. Compared to progesterone, which has an almost 'flat' molecular structure, dydrogesterone is bent due to a switch of the methyl group at carbon-10 from a beta position to an alpha position, and a switch of the hydrogen at carbon-9 from an alpha position to a beta position. ${ }^{(17)}$ The enhanced configuration of dydrogesterone results in its increased stability and, hence, higher bioavailability (i.e. $28 \%$ ) as compared to oral progesterone. ${ }^{(18)} \mathrm{A}$ few small studies that investigated the effectiveness of dydrogesterone in ARTs have found dydrogesterone to be effective for luteal phase support when compared to other types of progesterone. ${ }^{(9-11)}$ Chakravarty et $\mathrm{al}^{(9)}$ and Ganesh et $\mathrm{al}^{(10)}$ reported pregnancy rates of $22.8 \%$ and $28.7 \%$, respectively, when dydrogesterone $20 \mathrm{mg}$ per day was used for luteal phase support. Salehpour et al reported a pregnancy rate of $25 \%$ when dydrogesterone at $40 \mathrm{mg}$ per day was used for luteal phase support in ARTs. ${ }^{(11)}$ These three aforementioned studies did not find any significant differences between the pregnancy rates of women using dydrogesterone and those of women using vaginal progesterone. Only the study by Chakravarty et al reported on the rate of live births among the women using dydrogesterone; 19 out of 79 women (24.1\%) on dydrogesterone delivered successfully..$^{(9)}$ This live birth rate is similar to the rate of $27.7 \%$ found in the present study, which is also comparable to the average live birth rate of couples who conceive with ARTs in Singapore (i.e. 30\%). ${ }^{(19)}$

The rate of miscarriage for all pregnancies is estimated to range from $15 \%$ to $20 \%$. A retrospective analysis of all pregnancies conceived via ARTs in SGH from 1998 to 2003 revealed a miscarriage rate of $19.9 \% .{ }^{(20)}$ The miscarriage rate in the present study was $17.0 \%$, which is within the miscarriage rate for spontaneous pregnancies and is also the average miscarriage rate for ARTs done in our centre. Recurrent miscarriages, defined as three or more consecutive early pregnancy losses, affect $1 \%$ of couples. ${ }^{(21)} \mathrm{A}$ Cochrane review of studies that compared the effect of progesterone treatment on women with recurrent miscarriages to that of placebos or no treatment found that progesterone treatment significantly reduced miscarriage rates. ${ }^{(22)}$ In addition to progesterone's role in luteal phase support, studies have suggested that progesterone may have a role in promoting a shift toward anti-inflammatory cytokines, which help prevent maternal rejection of the immunologically foreign fetus. In normal pregnancies, progesterone-induced blocking factor is synthesised by activated lymphocytes in the presence of progesterone; this promotes the dominance of anti-inflammatory Th2 cytokines over inflammatory Th1 cytokines. ${ }^{(23,24)}$ Dydrogesterone binds almost exclusively to progesterone receptors, and since it is structurally similar to endogenous progesterone, it has been shown to induce a shift toward anti-inflammatory cytokines. ${ }^{(23)}$ In a randomised placebo-controlled trial that investigated the effect of dydrogesterone treatment on pregnancy outcomes among women with recurrent miscarriages, the risk of repeat miscarriage was found to be significantly higher (2.4 times) in the placebo group. ${ }^{(25)}$

A study that reviewed birth defects reported from 1977 to 2005 was not able to find any conclusive association between the use of dydrogesterone during pregnancy and congenital birth defects, due to the diversity of birth defects found. ${ }^{(12)}$ Similarly, there was no dominant type of fetal anomaly or any obvious pattern of abnormality among the seven cases of fetal anomalies detected in the present study. The fetal anomaly rate of $1.9 \%$ in our study was within the range reported in a meta-analysis of 19 studies, in which the rate of major congenital anomaly was $0 \%-9.5 \%$ for the IVF group, $1.1 \%-9.7 \%$ for the ICSI group and $0 \%-6.9 \%$ for the control group. ${ }^{(26)}$ Given the lack of observed association between dydrogesterone and the occurrence of birth defects, as well as the evidence that dydrogesterone has a protective effect against recurrent miscarriages, it is reasonable for dydrogesterone to be considered a viable option for luteal phase support in ARTs. One limitation of the present study was the lack of a control group, which prevented a comparison between the outcomes of intramuscular and vaginal progesterone use in the sample population.

In conclusion, while there has not been any evidence favouring a specific route of administration of progesterone for luteal phase support in ARTs in terms of outcomes, the oral route is favoured by women due to its ease of administration. The present study has shown that reasonable pregnancy and live birth rates can be achieved using dydrogesterone for luteal phase support in a large cohort of women. We did not observe any increased risk of fetal anomalies or miscarriages with the use of dydrogesterone in IVF. Thus, dydrogesterone can be considered for women who are not comfortable with injections or vaginal applications. Although current studies have evaluated the efficacy of dydrogesterone for luteal phase support with dosages ranging from $20 \mathrm{mg}$ to $40 \mathrm{mg}$ per day, none have examined the effect of different dosages of dydrogesterone on ART outcomes. Future large-scale, randomised controlled trials should be conducted to identify the optimal dosage of dydrogesterone for luteal phase support in ARTs.

\section{REFERENCES}

1. Daya S. Efficacy of progesterone support for pregnancy in women with recurrent miscarriage. A meta-analysis of controlled trials. Br J Obstet Gynaecol 1989; 96:275-80

2. Daya S. Issues in the etiology of recurrent spontaneous abortion. Curr Opin Obstet Gynecol 1994; 6:153-9.

3. Insler V. Corpus luteum defects. Curr Opin Obstet Gynecol 1992; 4:203-11.

4. Penzias AS. Luteal phase support. Fertil Steril 2002; 77:318-23.

5. Pritts EA, Atwood AK. Luteal phase support in infertility treatment: a metaanalysis of the randomized trials. Hum Reprod 2002; 17:2287-99.

6. van der Linden M, Buckingham K, Farquhar C, Kremer JA, Metwally M. Luteal phase support for assisted reproduction cycles. Cochrane Database Syst Rev 2011; (10):CD009154. 
7. Tavaniotou A, Smitz J, Bourgain C, Devroey P. Comparison between differen routes of progesterone administration as luteal phase support in infertility treatments. Hum Reprod Update 2000; 6:139-48.

8. Buvat J, Marcolin G, Guittard C, et al. [Luteal support after administration of an LHRH analog for in vitro fertilization. Superiority of vaginal progesterone in comparison with oral progesterone]. Presse Med 1990; 19:257. French.

9. Chakravarty BN, Shirazee HH, Dam P, et al. Oral dydrogesterone versus intravaginal micronised progesterone as luteal phase support in assisted reproductive technology (ART) cycles: results of a randomised study. J Steroid Biochem Mol Biol 2005; 97:416-20.

10. Ganesh A, Chakravorty N, Mukherjee R, et al. Comparison of oral dydrogestrone with progesterone gel and micronized progesterone for luteal support in 1,373 women undergoing in vitro fertilization: a randomized clinical study. Fertil Steril 2011; 95:1961-5.

11. Salehpour S, Tamimi M, Saharkhiz N. Comparison of oral dydrogesterone with suppository vaginal progesterone for luteal-phase support in in vitro fertilization (IVF): A randomized clinical trial. Iran J Reprod Med 2013; 11:913-8.

12. Queisser-Luft A. Dydrogesterone use during pregnancy: overview of birth defects reported since 1977. Early Hum Dev 2009; 85:375-7.

13. Ubaldi F, Bourgain C, Tournaye H, et al. Endometrial evaluation by aspiration biopsy on the day of oocyte retrieval in the embryo transfer cycles in patients with serum progesterone rise during the follicular phase. Fertil Steril 1997; 67:521-6.

14. Al Inizi ST, Asaad M, Schick J. Luteal phase support in in-vitro fertilization. Middle East Fertil Soc J 2006; 11:64-9.

15. Nillius SJ, Johansson ED. Plasma levels of progesterone after vaginal, rectal, or intramuscular administration of progesterone. Am J Obstet Gynecol 1971 110:470-7.
16. Simon JA, Robinson DE, Andrews MC, et al. The absorption of oral micronized progesterone: the effect of food, dose proportionality, and comparison with intramuscular progesterone. Fertil Steril 1993; 60:26-33.

17. Schindler AE, Campagnoli C, Druckmann R, et al. Classification and pharmacology of progestins. Maturitas 2008; 61:171-80.

18. Dydrogesterone. SGH ePharmacopoeia. Available at: http://online.lexi.com/ Ico/action/doc/retrieve/docid/multinat_f/4667159. Accessed July 29, 2014.

19. SingHealth. In-vitro fertilisation (IVF)/intra-cyctoplasmic sperm injection (ICSI). Available at: http://www.singhealth.com.sg/PatientCare/ ConditionsAndTreatments/Pages/IVF-In-Vitro-Fertilisation-ICSI-IntraCytoplasmic-Sperm.aspx. Accessed October 20, 2014

20. Rajesh H, Yap H, Wu YJ. Pregnancy outcomes from in-vitro fertilisation and intracytoplasmic sperm injection: a comparison. Singapore Med J 2006; 47:309-14.

21. Rai R, Regan L. Recurrent miscarriage. Lancet 2006; 368:601-11.

22. Haas DM, Ramsey PS. Progestogen for preventing miscarriage. Cochrane Database Syst Rev 2013; 10:CD003511.

23. Qureshi NS. Treatment options for threatened miscarriage. Maturitas 2009; 65 Suppl 1:S35-41.

24. Raghupathy R, Kalinka J. Cytokine imbalance in pregnancy complications and its modulation. Front Biosci 2008; 13:985-94.

25. Kumar A, Begum N, Prasad S, Aggarwal S, Sharma S. Oral dydrogesterone treatment during early pregnancy to prevent recurrent pregnancy loss and its role in modulation of cytokine production: a double-blind, randomized, parallel, placebo-controlled trial. Fertil Steril 2014; 102:1357-1363.e3.

26. Rimm AA, Katayama AC, Diaz M, Katayama KP. A meta-analysis of controlled studies comparing major malformation rates in IVF and ICSI infants with naturally conceived children. J Assist Reprod Genet 2004; 21:437-43. 